\title{
Hepatoblastoma in Developing Countries; Eight Years of Single Centre Experience
}

\author{
Sayed Abdelahamid1', Reham Abdelaziz Khedr' ${ }^{*}$, Madeeha El Wakeel2 ${ }^{2}$ Alaa Younes ${ }^{3}$, \\ Gehad Ahmed ${ }^{4}$, Naglaa Elkinaai ${ }^{5}$, Marwa Tantawy6, Hanafy Ahmed Hafez ${ }^{1}$
}

\author{
${ }^{1}$ Department of Pediatric Hematology/Oncology, National Cancer Institute, Cairo University, CCHE, Cairo, Egypt \\ ${ }^{2}$ Department of Radiodiagnosis, National Cancer Institute, Cairo University, CCHE, Cairo, Egypt \\ ${ }^{3}$ Department of Surgical Oncology, National Cancer Institute, Cairo University, CCHE, Cairo, Egypt \\ ${ }^{4}$ Department of Surgery, Faculty of Medicine, Helwan University, Cairo, Egypt \\ ${ }^{5}$ Department of Surgical Pathology, National Cancer Institute, Cairo University, Cairo, Egypt \\ ${ }^{6}$ Department of Clinical Research, CCHE, Cairo, Egypt \\ Email: ^riham.abdelaziz2003@gmail.com
}

How to cite this paper: Abdelahamid, S., Khedr, R.A., El Wakeel, M., Younes, A., Ahmed, G., Elkinaai, N., Tantawy, M. and Hafez, H.A. (2018) Hepatoblastoma in Developing Countries; Eight Years of Single Centre Experience. Journal of Cancer Therapy, 9, 793-806.

https://doi.org/10.4236/jct.2018.910065

Received: July 8, 2018

Accepted: October 6, 2018

Published: October 9, 2018

Copyright () 2018 by authors and Scientific Research Publishing Inc. This work is licensed under the Creative Commons Attribution International License (CC BY 4.0).

http://creativecommons.org/licenses/by/4.0/

\begin{abstract}
Background and objectives: Although hepatoblastoma ( $\mathrm{HB})$ is a rare childhood tumor and constitutes only $0.9 \%$ of all pediatric cancers, there was an obvious improvement in risk stratification and prognosis over the last two decades. This study aimed to assess the outcome of $\mathrm{HB}$ patients treated in our center and to investigate the impact of different prognostic factors on the survival of these patients. Patients and methods: This was a retrospective study included newly diagnosed patients with $\mathrm{HB}$ presented to the Children Cancer Hospital Egypt (CCHE 57357), from July 2007 to June 2015. Patient's data were analyzed for the clinical characteristics and survival outcome of the included patients. Results: One hundred twenty-four children were included during the study period with a median age of 14 months. The tumor was found occupying the entire liver in 25 patients (20\%); while it was confined to one lobe in $80 \%$ of them, portal vein thrombosis was diagnosed in 10 patients, and there were 30 patients (24\%) had metastatic disease at presentation. Only five patients (4\%) underwent primary surgical excision, and all of them were grossly excised (stage I); 77/119 (64.7\%) experienced delayed surgery after two to six courses (median, four courses) of C5VD and the overall resection rate was $66 \%$. There were 42 patients (35.3\%) failed to do surgical excision either because they still had evident metastatic disease with poor chemotherapy response, or because their tumor remained unresectable after six courses of chemotherapy. The 3-year event-free and overall survivals for the whole studied patients were $45.7 \%$ (95\% CI, 36.9\% - 56.7\%), and 66.7\% (95\% CI, 57.1\% - 77.8\%) respectively. The 3-year EFS and OS were significantly better in those who underwent surgical excision $(68.63 \%$ and $80.74 \%$
\end{abstract}


respectively, $\mathrm{P}$-value $<0.001)$. Also, the survival rates were significantly affected by the presence of metastatic disease at presentation, tumor stage and initial risk grouping of the studied patients. Conclusion: Surgical excision, tumor stage and COG risk grouping are the main prognostic variables affecting patients' outcome. Efforts to achieve resectability of the tumor either by advanced surgical techniques or by developing effective preoperative treatment, especially for advanced and metastatic disease, are mandatory.

\section{Keywords}

Hepatoblastoma, Risk Factors, Survival

\section{Introduction}

Hepatoblastoma (HB) is the most common malignant liver tumor in childhood and constitutes $0.9 \%$ of all pediatric cancers [1]. Numerous histological subtypes of $\mathrm{HB}$ have been identified; with different prognostic implications as patients with the small-cell undifferentiated subtype have a much worse prognosis than those with a purely fetal subtype, which has a good prognosis [2] [3]. Also, tumors with a macrotrabecular growth pattern may be associated with an unfavorable outcome [2].

Complete surgical resection is essential for the cure of hepatoblastoma, but about half of the patients with HB have an unresectable tumor at diagnosis [4]. With surgery only, the outcome of the patients with HB was dismal, with a $20 \%$ $30 \%$ chance of survival [5]. However, the introduction of neoadjuvant and adjuvant chemotherapy has dramatically improved the survival of these patients to $70 \%-80 \%$ [6]. Thus, chemotherapy is also an essential part of treatment for HB not only to facilitate surgical excision but also to prevent local recurrence and to control the metastatic disease.

Various chemotherapy agents have been used in the treatment of HB. The most common agents utilized have included cisplatin, carboplatin, doxorubicin, vincristine, 5-fluorouracil, ifosfamide, etoposide, and irinotecan in varying doses, schedules, and combinations. Cisplatin is typically included in most treatment regimens and has been considered to be the most active chemotherapy agent against $\mathrm{HB}$ [7] [8].

Numerous chemotherapeutic treatment strategies have been used by the international cooperative group studies in North America [COG], Europe (SIOPEL), and Japan which mainly determined by the surgical resectability, stage of disease, and the absence or presence of metastatic disease at diagnosis and they reported almost the same survival rates [9] [10] [11] [12].

The ongoing Children's Oncology Group (COG) study for patients with newly diagnosed HB, denoted AHEP0731, employs the risk-based approach based on stage, histology, resectability, and a-fetoprotein (AFP) level that stratify the patients into four risk categories; very low, low, intermediate and high risk groups 
with different therapeutic approaches [9].

Current research is now exploring how prognostic variables such as tumor pathology, biology, intravascular tumor invasion, positive microscopic surgical margins, and extent of tumor necrosis, alpha fetoprotein levels, and cytogenetics can be used to better delineate a more risk-based approach to the treatment of HB.

The aim of this study was to assess the outcome of hepatoblastoma patients treated in our center and to investigate the impact of different prognostic factors on the survival of these patients.

\section{Patients and Methods}

This was a retrospective study included newly diagnosed patients with hepatoblastoma presented to the Children Cancer Hospital Egypt (CCHE 57357), from July 2007 to June 2015. After approval by the local IRB committee, patient's data were collected from the medical records and analyzed for the followings.

\subsection{Investigations at Diagnosis}

Demographic details (age, sex, and weight) and complete physical findings were documented. Peripheral blood was analyzed for full blood count, electrolyte levels, hepatic, and renal functions. Coagulation profile and serum alpha-fetoprotein (AFP) concentrations were also measured. Pretreatment assessment of the extent of the primary tumor was done by abdominal ultrasound, computed tomography (CT) scan, and/or magnetic resonance imaging (MRI). CT scan of the chest assessed the metastatic spread. Hepatic angiography was done if needed before surgical intervention. Pathologic review of representative tissue slides was done for all patients enrolled in the study.

\subsection{Staging and Risk Stratification}

Surgical criteria determined disease staging after either surgical resection or biopsy and before the initiation of chemotherapy. Stage I, complete gross resection with clear margins; stage II, gross total resection with microscopic residual disease at the margins of resection; stage III, gross total resection with nodal involvement or tumor spill, or incomplete resection with gross residual intrahepatic disease; stage IV, metastatic disease with either complete or incomplete resection or biopsy. However, recently starting from 2013 the patients were staged according to the new COG staging system (Table 1), and stratified into four risk categories; very low, low, intermediate and high-risk groups which were derived from COG risk stratification system (Figure 1).

\subsection{Treatment}

According to the treatment protocols which were adopted from the North American guidelines, all patients were assessed initially for the possibility of primary surgical excision, but those with detectable metastatic disease or with 
tumors that were clearly unresectable on imaging at diagnosis, they started neoadjuvant chemotherapy. The standard chemotherapy regimen has been a combination of CDDP, VCR, and 5FU.

Tumor response was evaluated after each two chemotherapy cycles using abdominal CT, or MRI and lung radiographs or CT scan and tumor resectability was assessed. If feasible, partial hepatectomy was then performed, if the tumor was responding to chemotherapy but was still considered unresectable, a maximum of total six courses of chemotherapy was recommended before surgery.

\subsection{Definition of Response and Outcome Measures}

Using the revised RECIST guideline for response evaluation of solid tumors [13];

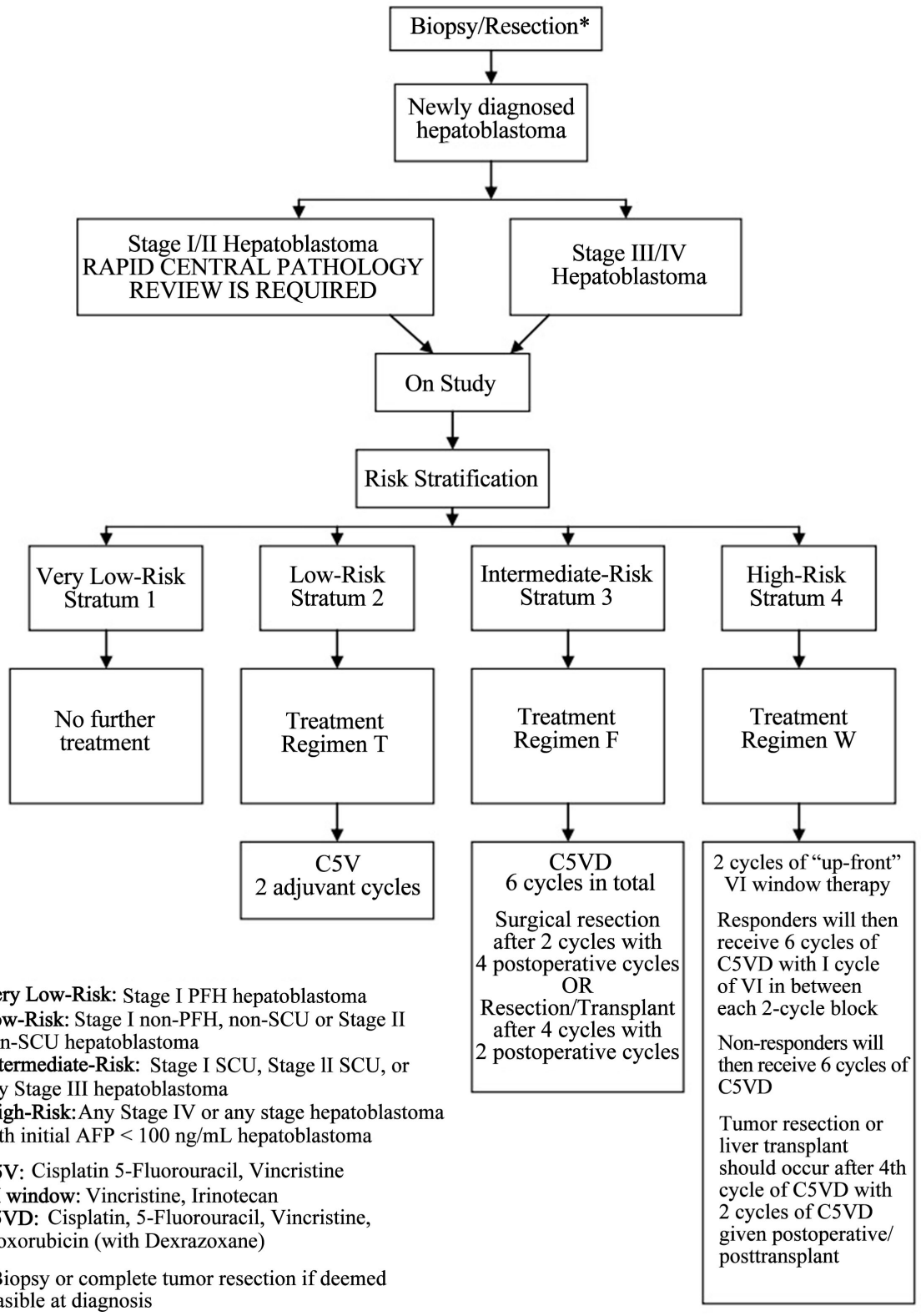

Figure 1. The revised COG staging system 2013. 
Table 1. COG staging system (2003).

\begin{tabular}{ll}
\hline Stage & \multicolumn{1}{c}{ Criteria } \\
Stage I & .The tumor is completely resected via wedge resection or lobectomy. \\
& .The tumor has PFH results. \\
& The AFP level is within reference range within 4 weeks of surgery. \\
IIA & .The tumor is completely resected. \\
.The tumor has histologic results other than PFH (UH). & IIB \\
.The tumor is completely resected. \\
Stage II \\
IIC findings are negative at time of diagnosis (no marker to follow). \\
.The tumor is completely resected with microscopic residual disease is present. \\
.The AFP level is elevated 4 weeks after resection. \\
Any of the following; \\
Stage III $\quad$. Ghe tumor is initially unresectable but is confined to one lobe of liver. \\
.Tumor ruptures or spills preoperatively or intra operatively. \\
.Regional lymph nodes are involved. \\
.Distant bone or lung metastasis is present.
\end{tabular}

the complete response was defined as the disappearance of all target lesions, with a normal serum AFP concentration. Partial response was defined as at least a $30 \%$ decrease in the sum of diameters of target lesions with a continued reduction in the serum AFP concentration. Progressive disease was at least a $20 \%$ increase in the sum of diameters of target lesions and/or the appearance of one or more new lesions. Stable disease was defined as neither sufficient shrinkage to qualify for PR nor sufficient increase to qualify for PD; the outcome measures included tumor response, surgical resectability rate, event-free survival and overall survival rates.

\subsection{Statistical Analysis}

Data obtained were summarized as Mean + SD (standard deviation) and percentages. Event-free survival (EFS) was defined as the period from the date of diagnosis until evidence of an event (progressive disease, relapse, or death) or last contact, whichever occurred first. Overall OS time was defined as the period from the date of diagnosis until death or last contact, whichever occurred first.

Correlations between different demographic factors of hepatoblastoma were done using Chi-square test or Fisher exact. P-values less than 0.05 were considered significant.

\section{Results}

This was a retrospective study included newly diagnosed patients with hepatoblastoma presented to the Children Cancer Hospital Egypt (CCHE 57357), from July 2007 to June 2015. One hundred forty children were included during the study period, 16 patients excluded from the analysis as they died too early before 
treatment initiation (Patients characteristics of the whole study cohort are summarized in Table 2).

The median age at diagnosis was 14 months (range: 0.5 month - 193 months) with a male to female ratio of 1.5:1. Pure fetal histology was the most common histological type which was identified in 49 patients (40\%), only three patients were diagnosed with undifferentiated small cell histology, and one patient had the epithelial macrotrabecular subtype.

Regarding tumor extension, the tumor was found occupying the entire liver in 25 patients (20\%), while it was confined to one lobe in $80 \%$ of them (70 patients on the right lobe and 29 patients on the left lobe), and portal vein thrombosis was diagnosed in 10 patients. There were 30 patients (24\%) had metastatic disease at presentation. Initial AFP level was above $100 \mathrm{ng} / \mathrm{mL}$ in 117 patients (94\%), while it was below $100 \mathrm{ng} / \mathrm{mL}$ in only seven patients (7\%). Of the whole study cohort and after surgical assessment for all non-metastatic patients, only 5 patients (4\%) underwent primary surgical excision and all of them were grossly excised (stage 1), while 119 (96\%) received preoperative chemotherapy because of advanced local disease and looked unresectable by the surgeon and/or presence of overt metastatic disease at presentation. For stage I patients, one had pure fetal histology and did not receive postoperative chemotherapy while the other four patients had different histological types and received low-risk protocol.

Table 2. Patients characteristics of the whole study cohort.

\begin{tabular}{ll}
\hline Characteristic & Patient no (\%) \\
\hline Age at diagnosis & \\
- -one year & $50(40.4 \%)$ \\
- >1 year and $<10$ years & $68(54.8 \%)$ \\
- $\geq 10$ years & $6(4.8 \%)$ \\
Gender & \\
- Males & $74(59.7 \%)$ \\
- Females & $50(40.3 \%)$ \\
Histological types & \\
- Pure fetal & $49(39.8 \%)$ \\
- Mixed epithelial/mesenchymal & $28(22.8 \%)$ \\
- Epithelial: combined embryonal and fetal & $25(20.3 \%)$ \\
- Epithelial, NOS & $16(13 \%)$ \\
- Small cell undifferentiated & $3(2.4 \%)$ \\
- Others & $3(2.4 \%)$ \\
Tumor Stage & \\
- Stage I & $5(4 \%)$ \\
- Stage III & $89(71.8 \%)$ \\
- Stage IV & $30(24.2 \%)$ \\
AFP level; ng/mL & \\
- Above 100 & $117(94 \%)$ \\
- Below 100 & $7(7 \%)$ \\
Risk Group & \\
- Very low risk & $1(0.8 \%)$ \\
- Low risk & $4(3.2 \%)$ \\
- Intermediate risk & $85(68.5 \%)$ \\
- High risk & $34(27.5 \%)$ \\
\hline
\end{tabular}


Of the 119 patients who had preoperative chemotherapy, 77 (64.7\%) experienced delayed surgery after two to six courses (median, four courses) of C5VD; thus the overall resection rate in our patients was $66 \%$. There were 42 patients (34\%) failed to do surgical excision either because they still had evident metastatic disease with poor chemotherapy response (18 patients). Also, the study reported 24 patients that remained unresectable after six courses of chemotherapy as they needed total hepatectomy, about $60 \%$ (14 cases) of them were candidates for orthotopic liver transplant which was not available in our center, while ten patients were not because of portal vein thrombosis.

Regarding treatment response and disease status at the end of therapy, 82 patients (66\%) had documented complete response, 11 patients (9\%) had partial response with residual unresectable disease, and 21 patients (17\%) showed progressive disease while there were 10 patients (8\%) died before end of therapy.

During the follow upperiod (average of 3 years), and of the 82 patients who achieved complete remission, we documented 19 relapses (23\%), 12 patients had local relapse in the liver and received salvage chemotherapy and surgical excision was done only for 4 patients, while 7 patients had metastatic relapse ( 6 cases with pulmonary metastasis and one case with brain metastasis).

\section{Outcome}

For all 124 patients, the 3-year event-free survival was $45.7 \%$ (95\% CI, 36.9\% $56.7 \%$ ), and the 3-year overall survival was $66.7 \%$ (95\% CI, 57.1\% - 77.8\%). (Survival outcomes in relation to different patients' parameters are detailed in Table 3).

The 3-year EFS and OS were significantly better in those who underwent surgical excision $(68.63 \%$ and $80.74 \%$ respectively, $\mathrm{P}$-value $<0.001)$. Also, the survival rates were significantly affected by the presence of metastatic disease at presentation, tumor stage, and initial risk grouping of the studied patients (Figure 2 and Figure 3). Initial AFP level (above or below $100 \mathrm{ng} / \mathrm{mL}$ ) was found to affect the OS but not the EFS, and there was no impact of the pathological types on the prognosis of the study patients.

\section{Discussion}

A defined treatment protocol for patients with HB was developed on 2000 and reported by the SIOP Liver Tumor Group (SIOPEL) which was consisting of neoadjuvant chemotherapy and surgical resection or early orthotopic transplant for those with too large tumors [14]. However, in the developing countries and Arab populations, there was a very limited and few data about the clinical behavior and outcome of this rare disease. Thus, based on the relatively big number of patients with $\mathrm{HB}$ in our center we tried to analyze our results using the staging system, risk stratification and treatment guidelines of the North American studies and the subsequent COG trials.

The survival outcome of the patients in this study was relatively lower than 
Table 3. Survival outcome in relation to different disease parameters.

\begin{tabular}{|c|c|c|c|c|c|c|}
\hline parameter & $\begin{array}{l}\text { EFS at } \\
3 \text {-ys }\end{array}$ & $95 \% \mathrm{CI}$ & $\mathrm{P}$-value & $\begin{array}{l}\text { OS at } \\
3-y s\end{array}$ & $95 \% \mathrm{CI}$ & P-value \\
\hline Whole patients & $45.7 \%$ & $36.9 \%-56.7 \%$ & & $66.7 \%$ & $57 \%-77.8 \%$ & \\
\hline \multicolumn{7}{|l|}{ Stage } \\
\hline$-I$ & $100 \%$ & & & $100 \%$ & & \\
\hline- III & $53.2 \%$ & $42.6 \%-66.4 \%$ & & $74.7 \%$ & $64.4 \%-86.6 \%$ & \\
\hline- IV & $17.8 \%$ & $8.1 \%-39.3 \%$ & $<0.001$ & $23.9 \%$ & $5.6 \%-100 \%$ & $<0.001$ \\
\hline \multicolumn{7}{|l|}{ Histology } \\
\hline - PFH & $51 \%$ & $36.7 \%-70.6 \%$ & & $81.3 \%$ & $70.3 \%-94 \%$ & \\
\hline - Combined & & & & & & \\
\hline (Fetal \& Embry) & $50.8 \%$ & $33.3 \%-77.4 \%$ & & $70 \%$ & $51 \%-94.8 \%$ & \\
\hline - Mixed & & & & & & \\
\hline (Epi \& mesen) & $55.5 \%$ & $39.5 \%-78 \%$ & 0.85 & $64 \%$ & $46 \%-89 \%$ & 0.5 \\
\hline \multicolumn{7}{|l|}{ Metastasis } \\
\hline - Yes & $18.5 \%$ & $8.4 \%-40.5 \%$ & & $24.8 \%$ & $5.8 \%-100 \%$ & \\
\hline - No & $54.8 \%$ & $44.5 \%-67.5 \%$ & $<0.001$ & $75.2 \%$ & $65.4 \%-86.5 \%$ & $<0.001$ \\
\hline \multicolumn{7}{|l|}{ AFP level } \\
\hline - Above 100 & $48.3 \%$ & & & $69 \%$ & & \\
\hline - Below 100 & $28.6 \%$ & & 0.1 & $38 \%$ & & 0.02 \\
\hline \multicolumn{7}{|l|}{ Surgery } \\
\hline - Yes & $68.6 \%$ & $58 \%-81.2 \%$ & & $80.7 \%$ & $70.6 \%-92.2 \%$ & \\
\hline - No & & & $<0.001$ & & & $<0.001$ \\
\hline \multicolumn{7}{|l|}{ Risk group } \\
\hline - LR & $100 \%$ & & & $100 \%$ & & \\
\hline - IR & $55.6 \%$ & & & $75.3 \%$ & & \\
\hline - HR & $19.4 \%$ & & $<0.001$ & $37.4 \%$ & & 0.001 \\
\hline
\end{tabular}

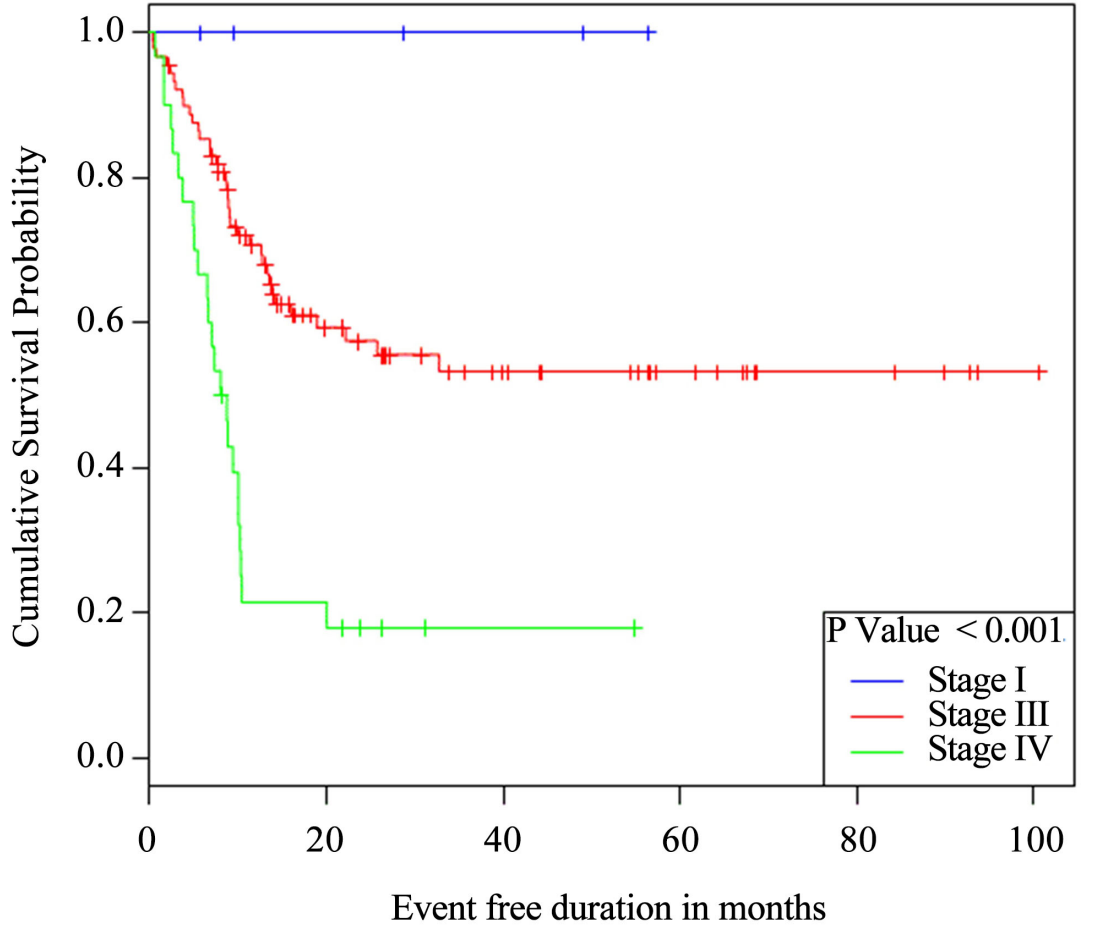

Figure 2. EFS of the whole cohort in relation to the COG Stage. 


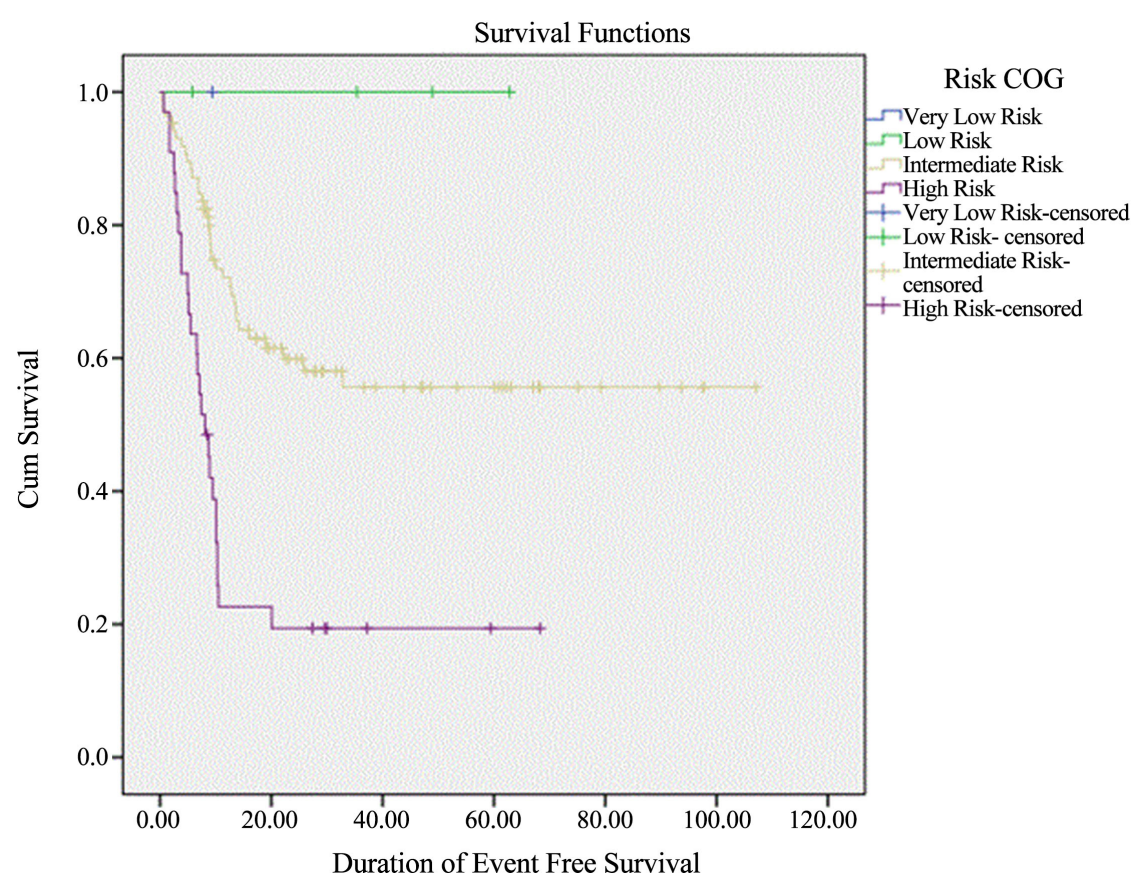

Figure 3. EFS in relation to the COG Risk stratification.

that reported in the other international studies like the North American study trials which were using CDDP/5-FU/VCR, as the standard chemotherapy regimen ( $>60 \%$ EFS and $>70 \%$ OS at five years) [3] [15]. Also the European studies (SIOPEL trails) reported much better survival rates, using different regimens including either CDDP alone or CDDP/DOX and intensified CDDP dose (66\% and $75 \%$ EFS and OS at 5 years respectively in SIOPEL- 1 and $76 \%$ and $83 \%$ at 3 years in SIOPEL-4) [6] [16].

Analysis of different factors that might affect the outcome in our patients' cohort, revealed that the outcome was significantly affected by initial tumor stage and presence of metastatic disease (100\%, 53\% and 17.8\% 3-year EFS for stage I, III and IV respectively) which was comparable to that reported by INT-0098 CCG study [3] (EFS at five years was $91 \%, 100 \%, 64 \%$, and $25 \%$ for stage I, II, III, IV respectively). And also the more recent POG trail P9645 showed similar findings with a relatively better survival of patients with metastatic disease (4 year EFS for stage I/II was $84 \%$, for stage III was $63 \%$, and for stage IV it was $50 \%)$ [15]. Worth mention that the percentage of the advanced stages in those studies is significantly lower than reported in our study, (42\% and $19 \%$ for stages III and IV respective) in INT-0098 CCG study [3], and (39\% and 10\% for stages III and IV respectively) in P9645 study [15] compared to ( $72 \%$ and $24 \%$ for stages III and IV respectively) in our study. So, it was evident that we had more patients with either inoperable disease or metastatic disease at presentation which subsequently affecting the outcome of the whole study cohort.

Adopting the COG risk stratification system, which was mainly based on initial tumor stage, histological subtypes and initial AFP level, we found significant 
correlation between the risk group and the survival outcome (100\%, 55\% and $19 \%$ EFS at 3 years for low risk, intermediate risk, and high risk respectively). This confirms the findings of the North American studies used the same risk stratification system $(100 \%$ for very low risk, $>90 \%$ for low risk compared to $60 \%-75 \%$ for standard risk and 30\% - 50\% for high risk) [3] [9] [17]. The SIOPEL group which was using somewhat different risk assessment based on the PRETEXT (PRE-treatment tumor extension) stage and any additional adverse features (metastasis, vascular involvement, extrahepatic disease, tumor rupture), demonstrated the similar observations. The data provided in SIOPEL-2, the 3-year EFS was 73\% for patients with standard-risk HB (PRETEXT I-III with no additional adverse features) and 48\% for patients with high-risk HB (PRETEXT-IV tumors and/or metastatic disease) [18], then the SIOPEL-3 reported 85\% and $65 \%$ EFS at 3 years for standard risk and high risk respectively [19]. Also a publication from the Japanese studies (JPLT-2), used Cisplatin/epirubicin as frontline therapy and salvage regimen consisting of ifosfamide, pirarubicin, etoposide, and carboplatin for those with inadequate response and/or high-risk disease (PRETEXT III/IV and metastatic disease), 5-year overall survival in non-metastatic HB was $100 \%$ for PRETEXT I, $87.1 \%$ for PRETEXT II, $89.7 \%$ for PRETEXT III and 78.3\% for PRETEXT IV [11]. Based upon these results, from the multi-institutional cooperative studies, risk-adapted therapy for patients with HB become an essential trend with tailoring the therapeutic dose intensity according to the patient risk and safely reduce the treatment intensity for those with low risk features to minimize the toxicity and intensify therapy for those with high risk disease to further improve their outcome.

Neoadjuvant chemotherapy in various combinations has been used successfully to allow for delayed complete resection of $\mathrm{HB}$, and approximately two-thirds of the initially unresectable tumors became resectable. In this study cohort, $64 \%$ of patients with initially unresectable tumors underwent complete surgical excision after preoperative chemotherapy, and the overall resection rate was $66 \%$ of the whole patients which was significantly lower than other international studies. In SIOPEL-3 trail, the resection rate was $95 \%$ for the standard risk patients [20], and it was $76.2 \%$ for high-risk patients either by partial hepatectomy $(55.6 \%)$ or by liver transplantation $(20.6 \%)$, those with non-metastatic PRETEXT-IV tumors, had $89 \%$ complete resection (50\% by partial hepatectomy and $39 \%$ by OLT) and the 3-year EFS of all patients who underwent OLT was 74\% [19]. Furthermore, the SIOPEL-4 that used intensified cisplatin-based chemotherapy followed by removal of all remaining tumor lesions if feasible (including liver transplantation and metastatectomy, if needed) for high-risk patients and reported $74 \%$ resection rate for the whole patients [16]. The lower resection rate in this study may be explained by lack of orthotopic liver transplant which was done in 14 patients (11.5\%), in addition to persistent overt metastatic disease after preoperative chemotherapy (14.5\%), so these data emphasize the role of OLT to further improve the resection rate especially for those with ad- 
vanced non-metastatic disease.

The treatment of metastatic hepatoblastoma remains a significant challenge, and this study showed a dismal outcome for those with metastatic disease, however, the SIOPEL-4 research showed much more encouraging results $(77 \%$ 3 -year event-free survival), and using intensification of dose-density of preoperative cisplatin to overcome the unfavorable outcome of those patients [16].

Relying on the fact that we were collecting pre-existing data, we were unable to implement the PRETEXT staging to all of the cases. Also due to the rarity of hepatoblastoma, multicenter and national cooperation should be made for further investigations and research with particular attention of Egypt because of the significant numbers of $\mathrm{HB}$ in a single center.

In conclusion, surgical excision, tumor stage and COG risk grouping are the main prognostic variables affecting the outcome of our patients. Further efforts should be made to achieve resectability of the tumor either by advanced surgical techniques including orthotropic liver transplant or by developing effective preoperative treatment, especially for the advanced and metastatic disease.

\section{Authors' Contribution}

I certify that all authors listed have participated sufficiently in the conception and design of this work, the analysis of the data (where applicable), as well as the writing of the manuscript, to take public responsibility for it as follows.

\begin{tabular}{cl}
\hline $\begin{array}{c}\text { Conceptualization } \\
\text { Software }\end{array}$ & All of the Authors \\
Validation & All of the Authors \\
Formal analysis & All of the Authors \\
Investigation & All of the Authors \\
Resources & All of the Authors \\
Data curation & All of the Authors \\
Writing-original draft & All of the Authors \\
Writing-review and editing & All of the Authors \\
Visualization & All of the Authors \\
Supervision & All of the Authors \\
Project administration & All of the Authors \\
All of the Authors
\end{tabular}

\section{Conflicts of Interest}

We declare no conflict of interest.

\section{References}

[1] Miller, R.W. and Young, J.L. (1995) Novakovic B: Childhood Cancer. Cancer, 75, 
395-405.

https://doi.org/10.1002/1097-0142(19950101)75:1+<395::AID-CNCR2820751321>3. $0 . \mathrm{CO} ; 2-\mathrm{W}$

[2] Zimmermann, A. (2005) The Emerging Family of Hepatoblastoma Tumours: From Ontogenesis to Oncogenesis. European Journal of Cancer, 41, 1503-1514.

https://doi.org/10.1016/j.ejca.2005.02.035

[3] Ortega, J.A., Douglass, E.C., Feusner, J.H., Reynolds, M., Quinn, J.J., Finegold, M.J., Haas, J.E., King, D.R., Liu-Mares, W., Sensel, M.G. and Krailo, M.D. (2000) Randomized Comparison of Cisplatin/Vincristine/Fluorouracil and Cisplatin/Continuous Infusion Doxorubicin for Treatment of Pediatric Hepatoblastoma: A Report from the Children's Cancer Group and the Pediatric Oncology Group. Journal of Clinical Oncology, 18, 2665-2675. https://doi.org/10.1200/JCO.2000.18.14.2665

[4] Katzenstein, H.M., London, W.B., Douglass, E.C., Reynolds, M., Plaschkes, J., Finegold, M.J. and Bowman, L.C. (2002) Treatment of Unresectable and Metastatic Hepatoblastoma: A Pediatric Oncology Group Phase II Study. Journal of Clinical Oncology, 20, 3438-3444. https://doi.org/10.1200/JCO.2002.07.400

[5] Exelby, P.R., Filler, R.M. and Grosfeld, J.L. (1975) Liver Tumors in Children in the Particular Reference to Hepatoblastoma and Hepatocellular Carcinoma: American Academy of Pediatrics Surgical Section Survey-1974. Journal of Pediatric Surgery, 10, 329-337. https://doi.org/10.1016/0022-3468(75)90095-0

[6] Brown, J., Perilongo, G., Shafford, E., Keeling, J., Pritchard, J., Brock, P., Dicks-Mireaux, C., Phillips, A., Vos, A. and Plaschkes, J. (2000) Pretreatment Prognostic Factors for Children with Hepatoblastoma-Results from the International Society of Paediatric Oncology (SIOP) Study SIOPEL. European Journal of Cancer, 36, 1418-1425. https://doi.org/10.1016/S0959-8049(00)00074-5

[7] Black, C.T., Cangir, A., Choroszy, M. and Andrassy, R.J. (1991) Marked Response to Preoperative High-Dose cis-Platinum in Children with Unresectable Hepatoblastoma. Journal of Pediatric Surgery, 26, 1070-1073. https://doi.org/10.1016/0022-3468(91)90676-K

[8] Douglass, E.C., Green, A.A., Wrenn, E., Champion, J., Shipp, M. and Pratt, C.B. (1985) Effective Cisplatin (DDP) Based Chemotherapy in the Treatment of Hepatoblastoma. Medical and Pediatric Oncology, 13, 187-190.

https://doi.org/10.1002/mpo.2950130405

[9] Malogolowkin, M.H., Katzenstein, H.M., Krailo, M. and Meyers, R.L. (2012) Treatment of Hepatoblastoma: The North American Cooperative Group Experience. Frontiers in Bioscience, 4, 1717-1723. https://doi.org/10.2741/e492

[10] Czauderna, P. (2012) Hepatoblastoma throughout SIOPEL Trials-Clinical Lessons Learnt. Frontiers in Bioscience, 4, 470-479. https://doi.org/10.2741/e392

[11] Hishiki, T., Matsunaga, T., Sasaki, F., Yano, M., Ida, K., Horie, H., Kondo, S., Watanabe, K.-I., Oue, T., Tajiri, T., Kamimatsuse, A., Ohnuma, N. and Hiyama, E. (2011) Outcome of Hepatoblastomas Treated Using the Japanese Study Group for Pediatric Liver Tumor (JPLT) Protocol-2: Report from the JPLT. Pediatric Surgery International, 27, 1-8. https://doi.org/10.1007/s00383-010-2708-0

[12] Watanabe, K. (2013) Current Chemotherapeutic Approaches for Hepatoblastoma. International Journal of Clinical Oncology, 18, 955-961.

https://doi.org/10.1007/s10147-013-0616-8

[13] Eisenhauer, E.A., Therasse, P., Bogaerts, J., Schwartz, L.H., Sargent, D., Ford, R., Dancey, J., Arbuck, S., Gwyther, S., Mooney, M., Rubinstein, L., Shankar, L., Dodd, L., Kaplan, R., Lacombe, D. and Verweij, J. (2009) New Response Evaluation Crite- 
ria in Solid Tumours: Revised RECIST Guideline (Version 1.1). European Journal of Cancer, 45, 228-247. https://doi.org/10.1016/j.ejca.2008.10.026

[14] Pritchard, J., Brown, J., Shafford, E., Perilongo, G., Brock, P., Dicks-Mireaux, C., Keeling, J., Phillips, A., Vos, A. and Plaschkes, J. (2000) Cisplatin, Doxorubicin, and Delayed Surgery for Childhood Hepatoblastoma: A Successful Approach-Results of the First Prospective Study of the International Society of Pediatric Oncology. Journal of Clinical Oncology, 18, 3819-3828. https://doi.org/10.1200/JCO.2000.18.22.3819

[15] Malogolowkin, M.H., Katzenstein, H., Krailo, M.D., Chen, Z., Bowman, L., Reynolds, M., Finegold, M., Greffe, B., Rowland, J., Newman, K., Womer, R.B., London, W.B. and Castleberry, R.P. (2006) Intensified Platinum Therapy Is an Ineffective Strategy for Improving Outcome in Pediatric Patients with Advanced Hepatoblastoma. Journal of Clinical Oncology, 24, 2879-2884.

https://doi.org/10.1200/JCO.2005.02.6013

[16] Zsiros, J., Brugieres, L., Brock, P., Roebuck, D., Maibach, R., Zimmermann, A., Childs, M., Pariente, D., Laithier, V., Otte, J.-B., Branchereau, S., Aronson, D., Rangaswami, A., Ronghe, M., Casanova, M., Sullivan, M., Morland, B., Czauderna, P., Perilongo, G., International Childhood Liver Tumours Strategy Group (SIOPEL) (2013) Dose-Dense Cisplatin-Based Chemotherapy and Surgery for Children with High-Risk Hepatoblastoma (SIOPEL-4): A Prospective, Single-Arm, Feasibility Study. The Lancet Oncology, 14, 834-842. https://doi.org/10.1016/S1470-2045(13)70272-9

[17] Malogolowkin, M.H., Katzenstein, H.M., Meyers, R.L., Krailo, M.D., Rowland, J.M., Haas, J. and Finegold, M.J. (2011) Complete Surgical Resection Is Curative for Children with Hepatoblastoma with Pure Fetal Histology: A Report from the Children's Oncology Group. Journal of Clinical Oncology, 29, 3301-3306. https://doi.org/10.1200/JCO.2010.29.3837

[18] Perilongo, G., Shafford, E., Maibach, R., Aronson, D., Brugières, L., Brock, P., Childs, M., Czauderna, P., MacKinlay, G., Otte, J.B., Pritchard, J., Rondelli, R., Scopinaro, M., Staalman, C., Plaschkes, J. and International Society of Paediatric Oncology-SIOPEL 2 (2004) Risk-Adapted Treatment for Childhood Hepatoblastoma Final Report of the Second Study of the International Society of Paediatric Oncology-SIOPEL 2. European Journal of Cancer, 40, 411-421. https://doi.org/10.1016/j.ejca.2003.06.003

[19] Zsíros, J., Maibach, R., Shafford, E., Brugieres, L., Brock, P., Czauderna, P., Roebuck, D., Childs, M., Zimmermann, A., Laithier, V., Otte, J.-B., de Camargo, B., MacKinlay, G., Scopinaro, M., Aronson, D., Plaschkes, J. and Perilongo, G. (2010) Successful Treatment of Childhood High-Risk Hepatoblastoma with Dose-Intensive Multiagent Chemotherapy and Surgery: Final Results of the SIOPEL-3HR Study. Journal of Clinical Oncology, 28, 2584-2590. https://doi.org/10.1200/JCO.2009.22.4857

[20] Perilongo, G., Maibach, R., Shafford, E., Brugieres, L., Brock, P., Morland, B., de Camargo, B., Zsiros, J., Roebuck, D., Zimmermann, A., Aronson, D., Childs, M., Widing, E., Laithier, V., Plaschkes, J., Pritchard, J., Scopinaro, M., MacKinlay, G. and Czauderna, P. (2009) Cisplatin versus Cisplatin plus Doxorubicin for Standard-Risk Hepatoblastoma. The New England Journal of Medicine, 361, 1662-1670. https://doi.org/10.1056/NEJMoa0810613 


\section{Abbreviations}

Hepatoblastoma

COG
HB

Children Oncology Group 